\section{Perfusion Fixation of Research Animals}

\author{
Charles W. Scouten, ${ }^{*}$ Ryan O’Connor, ${ }^{* *}$ \\ and Miles Cunningham** \\ ${ }^{*}$ MyNeuroLab.com \\ St Louis, MO \\ cwscouten@myneurolab.com \\ ${ }^{* *}$ McLean Hospital of Harvard University \\ Belmont, $M A$ \\ mcunningham@mclean.harvard.edu
}

Perfusion, washing out blood and using the open vascular channel to infuse fixative, is the standard first step of preparing animal tissues for later examination under a microscope. The rapid and homogeneous fixation resulting provides an advantage over immersion fixation that is usually used for biopsy and clinical tissue samples (Cammermeyer, 1960, Garman, 1990). A disadvantage is that brain tissue, and probably other soft tissues, prepared by perfusion has no retained extracellular space post perfusion, although living brain has about $20 \%$ extracellular space (discussed in Cragg, 1980). Perfused brain is also about $20 \%$ shrunk in whole organ volume from living brain size. The shrinkage is uneven, and distorts the relationships of structures. It is possible to avoid the organ shrinkage and distortion that follows a traditional perfusion, with little additional effort. This is a discussion of how to optimize your perfusion results, why the shrinkage occurs, and how it can be avoided.

Given the importance of perfusion as the starting point of almost all of animal histology, the nuisance of red blood cells, and poor tissue working quality following poor perfusion, it gets surprisingly little attention in the methods section of published papers. Any red blood cells remaining in the brain affect the quality and intensity of labeling for HRP reactions and autofluoresce to obscure fluorescent labels. Length of fixative exposure affects any immune reaction. Perfusion pressures or flow rates, tubing diameters, cannula gauge, and flow rates vary among labs, but commonly are not reported.

There are 3 compartments in brain to think about: The vascular system, the extracellular fluid (ECF) and the cytosol, or intracellular fluid (ICF). Fluid components are different between the 3 compartments, especially in brain, with the blood/brain barrier. Cells can swell or shrink by exchanging water between ECF and ICF, without necessarily changing whole organ size or shape. Flow into ECF from the capillaries would necessarily cause swelling of whole organ. Chronic high blood pressure can cause whole organ swelling (edema) from just hydrostatic fluid pressure.

\section{Several terms should be defined to refresh memories:}

Osmolarity is a physics term describing the number of particles in a fluid. Osmolarity can be calculated from the molecular weight of the compound in the water, and grams of it added, and the degree to which it breaks up into dissociated ions. Intracellular fluid of living cells usually fluctuates about 330 milliMolar (mM) osmolarity. By contrast, four percent formaldehyde in water, commonly used in fixation, is about $1400 \mathrm{mM}$ osmolarity.

Tonicity is a more complicated relative term. It refers to particle distribution across a semi-permeable membrane, such as a cell membrane. Water will move across the membrane to equalize particle tonicity, even if that causes cellular swelling or shriveling.
- Only particles that can not freely cross the membrane contribute to tonicity, and only to the degree that they are unevenly distributed. Urea added to the cellular environment would instantly be distributed equally inside and outside the cell, and raise the osmolarity both places, but have no effect on tonicity and so no effect on water movements (http://physioweb.med. uvm.edu/bodyfluids/calculat.htm). Viewed in another way, a $340 \mathrm{mM}$ solution of urea would be as hypotonic as distilled water.

- Formaldehyde is soluble in benzene and chloroform, and therefore would be expected to cross cell membranes relatively freely (Cragg, 1980, Richard Thrift on www.biotech.ufl. edu/EM/data/osmos.html). Although it has high osmolarity in normal use ( $1400 \mathrm{mM}$ by itself, without the buffer) it therefore contributes only transiently to tonicity.

Isotonic Refers to a fluid that has equal tonicity to the fluid inside a living cell, and will not cause water exchange through the membrane. Since internal tonicity begins to change rapidly at onset of anoxia during a perfusion, and membrane permeability begins to increase with fixation, the cells internal fluids are no longer "isotonic" after the perfusion begins. The concept is irrelevant shortly after the onset of perfusion.

Cellular Pumps Cell membranes are permeable to sodium, but energy-using proteins on the membrane surface continuously pump sodium out, until it is distributed about 10:1 in ECF:ICF, and effects tonicity as if the membrane were impermeable to it. Sodium is therefore a big contributor to tonicity in the normal environment. There are several other active pumps in the cell, moving other molecules in or out of the cell. Anything that alters cellular metabolism - anoxia, lack of energy supply, chemical environment, toxins, fixatives, thermal shock, etc. will slow or stop the pumps, and sodium will flow in unchecked until evenly distributed ECF:ICF. Since the cell is in normal tonic balance with the sodium pumped out, its entrance will raise internal relative to external osmolarity. Water will need to flow into the cell to achieve balance. There are other pumps, e.g. potassium is pumped into the cell, and will flow out under challenge conditions, reducing the impact of sodium changes on osmolarity and tonicity. However, the short term result of most metabolic challenge is cellular swelling, suggesting that sodium and other cell entrants overbalance exits. Conversely, the long term result of fixation is whole organ shrinkage, not necessarily predicted by either cellular swelling or cellular shrinkage due to fluid movements between ECS and ICS. This mechanism needs to be explained.

Autolysis Cells, especially neurons, when challenged by, e.g. anoxia, very soon begin to break down internally. Large molecules are broken down into constituents. This results in a proliferation of particles, raising internal tonicity and also causing water to enter the cell from the ECF and probably capillaries as well. This contributes to the cellular swelling usually seen on cell death.

Fluid Dynamics of Perfusion As sodium flows in and autolysis starts, the cell swells. However, many ions are moving out of cells down a concentration gradient into flowing prewash (usually saline or PBS)) solution. Potassium coming out would enrich the $\mathrm{ECF}$, toward balance, but the ECF is being continuously replaced by flowing prewash solution. Ions and particles capable of crossing the membrane that are not represented in the prewash solution would be steadily leaving the cells down the concentration gradient. 


\section{The Next Step...}

\section{Innovative Solutions for Nano-Technology}

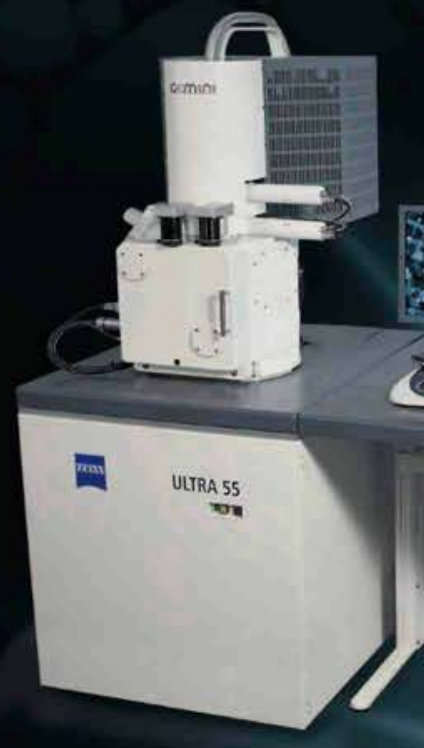

\section{ULTRA FESEM}

An extension of our leading SUPRATM series with new In-column EsB detector for superb high resolution imaging and unmatched compositional data.

Enabling the Nano-Age World ${ }^{\circ}$

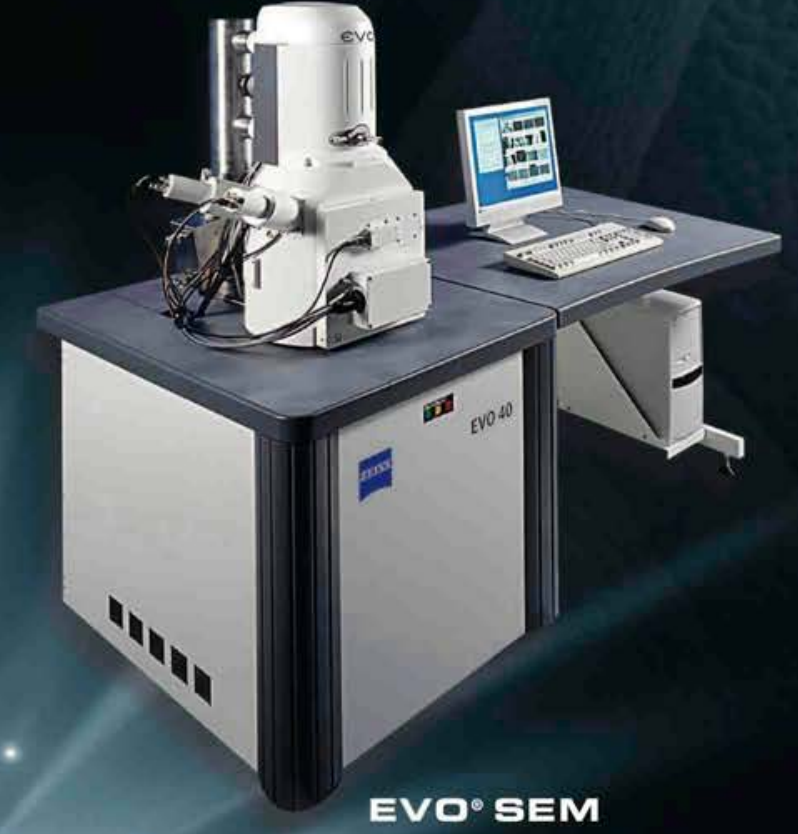

Tungsten or $\mathrm{LaB}_{6}$ system with superior imaging quality, class leading analytical capabilities and extened pressure option.

\section{LIBRA'EFTEM} Unique In-column OMEGA filter combined with Koehler illumination for unrivalled flexibility in imaging and analysis.
Carl Zeiss SMT Inc

Nano Technology Systems Division One Zeiss Drive

Thornwood, New York 10594 USA
Tel. $+1914 / 7477700$

Fax $+1914 / 6817443$

info-usa@smt.zeiss.com

www.smt.zeiss.com/nts 
With the start of fixation, Aldyhydes increase the permeability of cell membranes, further driving this effect, letting more particles out into the flowing fixative solution.

Once anoxia starts, or fixative arrives, the internal osmolarity of the cell begins to depart from "isotonic" due to many influences. The first is probably a large surge in osmolarity and tonicity due to sodium in-rush and internal autolysis. This accounts for the usual observation of cellular swelling. Later, the cell is probably depleted in most ions and particles as they leave the cell down concentration gradients to the perfusion solutions, and as the fixative makes the membrane permeable to more and more particles. At that late perfusion stage, osmolarity and tonicity are probably low inside the cell relative to living "isotonicity".

\section{Control of Pressure vs. Flow Rate for Perfusion}

Gravity flow or a peristaltic pump are the common controlled means of applying pressure to the perfusion fluid. Gravity provides a constant pressure, while a peristaltic pump provides a constant flow rate regardless of resistance. The flow rate and pressure can not both be controlled, of course. The flow rate equals the pressure divided by the cardiovascular resistance. Cardiovascular resistance is highly variable between and within species, while blood pressure is comparatively very consistent (Short, 1987, Green, 1979). Therefore, it is easier to select a pressure that is appropriate across species and individuals than it is to select a flow rate. The following is a table of average blood pressure for several species (systolic/diastolic) (Green, 1979).

$\begin{array}{llllll}\text { mice } & 113 / 81 & \text { rats } & 116 / 90 & \text { hamster } & 150 / 110 \\ \text { rabbit } & 110 / 80 & \text { dog } & 112 / 56 & \text { cat } & 120 / 75 \\ \text { baboon } & 148 / 100 & \text { rhesus } & 160 / 127 & \text { pig } & 170 / 108 .\end{array}$

baboon $148 / 100$ rhesus $160 / 127$ pig 170/108

Consider the extreme case of a flow rate used in rats being applied in a pig. Pressure would be minimal. The prewash fluid would find a few channels through, and trickle out, but blood washout would be very poor. Consequently, fixative would not flow into many blocked capillaries, and autolysis would be in progress before fixative reached the tissue by diffusion. Clearly, the flow rate selected must take into account the cardiovascular resistance of the animal, and be adjusted to generate a reasonable pressure.

Now consider applying $200 \mathrm{~mm} \mathrm{Hg}$ fixed pressure in either species. This is well above average blood pressure in both species, but in a range that can occur naturally without immediate damage. The resulting flow rate will be dramatically greater in the pig than in the rat, but washout and fixative distribution will be excellent in both cases. Capillaries are opened and blood pushed out by pressure, not flow rate. Fixative only enters capillaries not blocked by blood cells.

Cardiovascular resistance will vary widely between species, genders, strains, and individuals within strains. It will depend on previous exercise, body weight, fat percentage, and other variables. A fixed perfusion flow rate in the physiological range will thus result in systematic bias, by gender, weight, or any variable influencing cardiovascular resistance, in the quality of perfusion achieved. How much should the flow rate be adjusted to compensate for cardiovascular resistance differences for a rat 100 grams heavier than another rat?

Therefore, it seems apparent that more reproducible results will be obtained with a procedure that applies a known and controllable pressure to the fluid used, rather than a known flow rate, if there is any variance in cardiovascular resistance between the subject animals. What pressure should be selected?

\section{Traditional Gravity Perfusion Apparatus}

Although the gravity pressure apparatus commonly used for perfusion is simple, it is surprising that no commercial version has previously been offered for whole animal perfusion, given the ubiquity of the procedure in animal research. No manual and very little journal information offers directions for reservoir height, tubing size, or needle gauge. Every lab procures parts and assembles their own apparatus, with differing ideas of what may be important. Commonly, two containers with tubing attached to the bottom are set on a shelf at an arbitrary height. Both bottles are connected to the upper arms of a Y connector by tubing. Tubing clamps between the bottles and the $\mathrm{Y}$ connector enable control of which fluid is flowing. Tubing from the lower arm of the "Y" connector is connected to a plastic syringe barrel from which the flange has been removed. A gavage needle is installed on the other end of the syringe barrel.

\section{The Math}

In a gravity perfusion, the pressure of perfusate entering the animal's vasculature is determined by bottle height, minus pressure lost to the tubing and the needle's resistance to flow. The animal's cardiovascular resistance, not the apparatus, should be the variable that determines the flow rate. Commonly, but variably, the bottles are 25-40 inches above the work area on which the animal lies. Gravity pressure on an optimistic 40 inches of water translates to $76 \mathrm{~mm} \mathrm{Hg}$, a relatively low constant pressure for the mammalian vascular system. Even at physiological pressures, capillaries clog and unclog regularly. Some pressure is lost in flow through the tubing and more through the needle, which may offer as much resistance to flow as the open vascular system (which would mean the animal might get as little as half the gravity pressure, e.g., 38 $\mathrm{mm} \mathrm{Hg}$ pressure drop across the vascular system). This may account for the commonly observed inefficiency in removing red blood cells from brain capillaries. This common system does not provide enough pressure to fully wash out the blood. A pressure of $200 \mathrm{mmHg}$ would require that the bottles be $9 \mathrm{ft}$. above the animal, not possible in most lab spaces. Many researchers use a peristaltic pump to drive the fluids, rather than gravity. Variable pump speeds and thus flow rates and pressures are used and are not standardized. Flow rate is controlled rather than pressure. Optimal flow rate will depend on species, age, gender, cardiovascular condition, and any other factor that can affect cardiovascular resistance, but cannot be readily calculated. Thus, there are unaddressed issues with commonly used methods of perfusion.

\section{Soft Tissue Shrinkage}

On the electron microscopic level, brain cells and processes perfused with standard methods as described above reliably appear in apposition with each other, with no extracellular space. Several lines of evidence including resistance studies, cell counts against living volume, and electron microscopy of snap frozen tissue (van Harreveld and Steiner, 1970) show that the living brain is about $20 \%$ extracellular space (reviewed by Van Harreveld, 1972). In perfused and fixed tissue, using traditional protocols, this space is absent, and the brain is reduced in volume by about $20 \%$. This was and is accepted by most scientists as an unavoidable consequence of tissue processing, and is described as such in the stereotaxic atlases "This method needs some comment. It inevitably implies shrinkage caused by embedding and staining. Shrinkage can not be equalized by enlargement because, for physical reasons, the extent of 


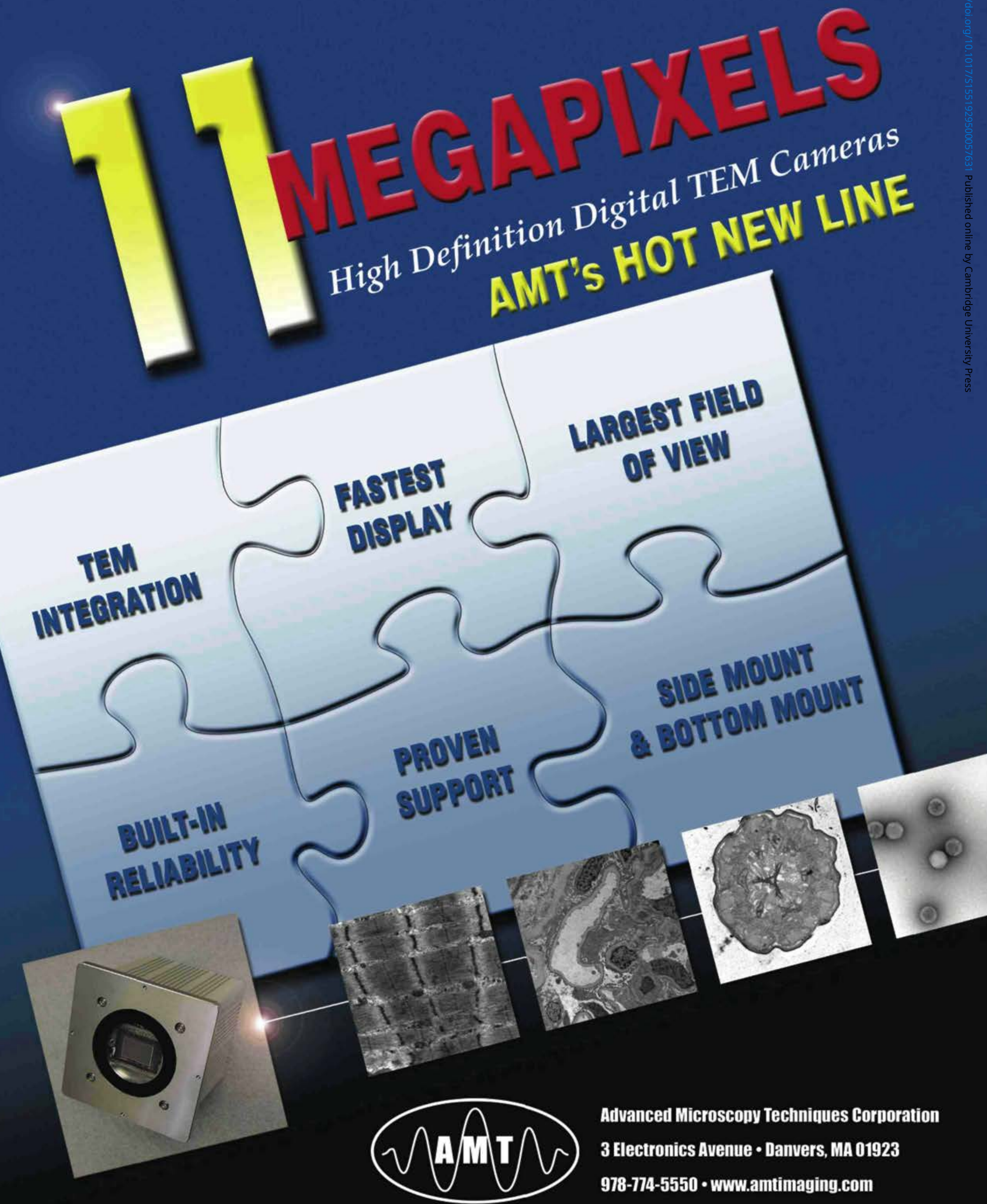


shrinkage differs in the various constituents of the brain" (König and Klipple, 1967). Later sections in König and Klipple made it clear the formaldehyde was the part of "embedding and staining" that caused the shrinkage. As a result, König and Klipple, 1967 could not provide accurate stereotaxic coordinates that could be applied to living brain.

The brain atlas by Paxinos and Watson (1998), avoided this problem by working only with fresh frozen tissue, and not fixing. Of course, many histological reactions do not work with fresh tissue. Proteins are coagulated by the dehydrating and mounting process. Nissl stains for purposes of gross anatomy do work fine on unfixed tissue. The widest distance across any coronal section of whole brain is about $13 \mathrm{~mm}$ in König and Klipple, while this distance is $16 \mathrm{~mm}$ in Paxinos and Watson (1998). Although there are age and weight differences, the brain size of adult rats changes only slightly with advancing age (Paxinos G, Watson C, Pennisi M, Topple A, 1985 ) and cannot account for this discrepancy. Rather, the $20 \%$ difference may be attributed to differences in tissue preparation for the König and Klippel (1967) atlas vs. the Paxinos and Watson (1998) atlas. The perception that fixation induced shrinkage is inevitable is widely held to this day, and is the prevailing wisdom (http://www.mbl.org/atlas247/atlas247_start.html) It is not inevitable, it can be avoided.

Preserving the Extracellular Space, Avoiding Shrinkage and Distortion

We will propose a model that the cellular swelling upon exposure to fixative, probably due mostly to movement of sodium from ECF to ICF due to offset of the cellular pumps, and to internal autolysis contributing particles to the ICF, results in loss of the extracellular space due to the ECF entering the cells. We further propose that once lost, the extracellular space does not reopen. The membranes adhere and stay together for unknown reasons. Outflow of ions and particles down the gradient from the cells into the flowing perfusion fluid eventually depletes the ICF, and the cells become internally hypotonic. Water flows out into the capillary fluid, and the cell returns to normal size or smaller. Now, however, each shrinking cell pulls its neighbor in with it, rather than restoring the extracellular space. The $20 \%$ shrinkage seen with formaldehyde or glutaraldehyde fixation at any concentration or exposure time is thus the volume of missing extracellular space.

Brian Cragg (1980) reasoned that removing ionic particles, especially sodium, from the extracellular spaces, and replacing them with an isotonic solution that could not cross the blood brain barrier, would prevent the swelling and thus spare the extracellular space. Ordinary sucrose is a substance that does not cross cell membranes. Perfusing with an isotonic solution of sucrose, and replacing the ECF with it, should hold the extracellualar space open and prevent some of the cellular swelling. Sodium and other ions could not move into the cell when the pumps stopped if they were not present in the ECF.

Any fluid, including sucrose, which cannot cross cell membranes also cannot cross the blood-brain barrier during perfusion, and thus cannot replace the extracellular fluid in brain. However, the blood brain barrier may be breached by pressure, without rupturing blood vessels. Cragg (1980) employed a peristaltic pump, a pressure gauge, and manual regulation of the flow rate in order to deliver a pressure of $300 \mathrm{~mm} \mathrm{Hg}$, which will force sucrose across the blood brain barrier (Rappoport, 1976).
The continuous flow of sucrose would carry away particles moving out of the cell, thus anchoring the extracellular fluid at 330 $\mathrm{mM}$ and free of any ions. No sodium was available to run in. The period of autolysis was shortened by a shorter washout period with the high pressure. Only autolysis was still promoting cellular swelling. Everything inside the cell had a steep concentration gradient to the outside. Fixative was added to the sucrose solution after initial washout (which should only transiently affect its tonicity). This worked too well, and left the cells somewhat shriveled. Not only was there still extracellular space, there was an excessive amount of extracellular space in the fixed tissue.

From this, we may infer that the intracellular tonicity had moved below that of normal living tissue in his conditions before size fixation was achieved. This seems a reasonable inference, since all the particles in the cell were now not represented outside the cell, and so all gradients were steep to the outside from the onset of perfusion. Ions that could cross the membrane left the cell down the gradients, and were washed away by fluid flow. Internal osmolarity below external meant that water must leave the cell, not enter it. Further, this seems to have been achieved in time to prevent the initial burst of swelling, or the extracellular spaces would have closed. Cragg does not mention anything about whole organ size or if shrinkage occurred.

A solution of total osmolarity of $280 \mathrm{mM}$ (somewhat hypotonic) was made up of $140 \mathrm{mM}$ sucrose and $140 \mathrm{mOsm}$ phosphate buffer. Using this as both the prewash and the base for fixative, and high pressure for the prewash, Cragg achieved perfused brain tissue with a $20 \%$ extracellular space. Cragg did not describe the effect of either procedure on the gross morphology of the brain, on overall shrinkage, on red blood cell retention, or histological analysis at the light microscopic level.

By as yet unknown mechanisms, the traditional process of perfusion fixation results in uneven whole organ shrinkage of soft tissue by about $20 \%$, which is also the normal amount of now missing extracellular space. The possibility that the cells return to normal size as the fixed membrane becomes fully permeable and fluid flows out, but that the whole organ collapses into the extracellular spaces, suggests itself. If so, somehow cells stay stuck against each other as they reduce in size. The unevenness of the shrinkage results in distortion of tissue gross anatomy, and has been a continuing thorn for neuroscientists studying anatomical relationships and positions. Preventing this shrinkage, at least for some research projects, would be very helpful. It would also make possible castings of fixed brains that would fit fresh brains. The brains produced by Cragg, with normal extracellular space, may not have been shrunken.

An alternative solution could have been a lowered osmolarity of sucrose solution for the second (fixative) stage. That is, one could add some ions to the sucrose solution, and thus raise the internal osmolarity of cells as Cragg did, or leave the ions out, wash them away, and make the second solution "hypotonic" relative to living cells, but to match the state of the fixed cells. Either procedure should inflate the cells to the right level. As long as the initial burst of swelling is avoided, the extracellular space would be retained and can be increased, maintained or reduced during fixation by tonicity of the flowing fluid relative to what remains in the cells.

\section{Tissue Other than Brain}

The sodium pump, autolysis, and the effects on tonicity are 


\section{Analytical Electron Microscopist}

Lawrence Berkeley National Laboratory (LBNL) is located in the San Francisco Bay Area on a 200 -acre site in the hills above the University of California's Berkeley campus and is managed by the University of Califomia. A leader in science and engineering research for more than 70 years, Berkeley Lab is the oldest of the U.S. Department of Energy's National Laboratories.

The National Center for Electron Microscopy (NCEM) at LBNL has an immediate opening for an outstanding materials scientist and electron microscopist to lead its Analytical Electron Microscopy research program. NCEM is a national user facility for electron beam microcharacterization of materials. The facility operates state-of-the-art electron microscopes and develops/applies advanced techniques and instrumentation for materials characterization. Learn more at http://ncem.lbl.gov

The incumbent will provide high-level expertise and conduct original research in electron beam microanalysis as an advanced tool for materials research and a subject of technique development. He/she will lead the operation, user program, and further development of advanced analytical electron microscopes and their application to significant scientific problems. It will be necessary to initiate collaborative projects with internal and external investigators, conceive novel experiments, and develop new spectral microscopy techniques and instrumentation. The candidate will also take a major role in the Transmission Electron Aberration-corrected Microscope (TEAM) project with expertise in aberration corrected STEM microscopy and analysis.

The successful candidate will have exceptional expertise in STEM/TEM imaging, fineprobe microanalysis, and materials research, with a strong publication record in the development and application of EELS and X-ray spectroscopies. He/she should be familiar with aberration correcting electron optics, and able to design, test, and apply novel techniques, instrumentation, or software for electron beam microanalysis. A Ph D. or equivalent experience in the physical sciences field is required. Expertise with soft/hard matter interfaces, computing, or modeling is desirable.

This is an indefinite career appointment. Applicants are requested to apply online at http://jobs.Ibl.gov, to job requisition \#018874. In addition, the applicant must submit a curriculum vitae, list of publications, statement of research interests, and the names of at least four references to NCEM Search Committee Job MS018874, Materials Sciences Division, MS 72-150, Lawrence Berkeley National Laboratory, Cyclotron Road, Berkeley, CA 94720

LBNL is an AAVEO employer committed to developing a safe and diverse workforce. For more information about Berkeley Lab and its programs, visit www.lbl.gov
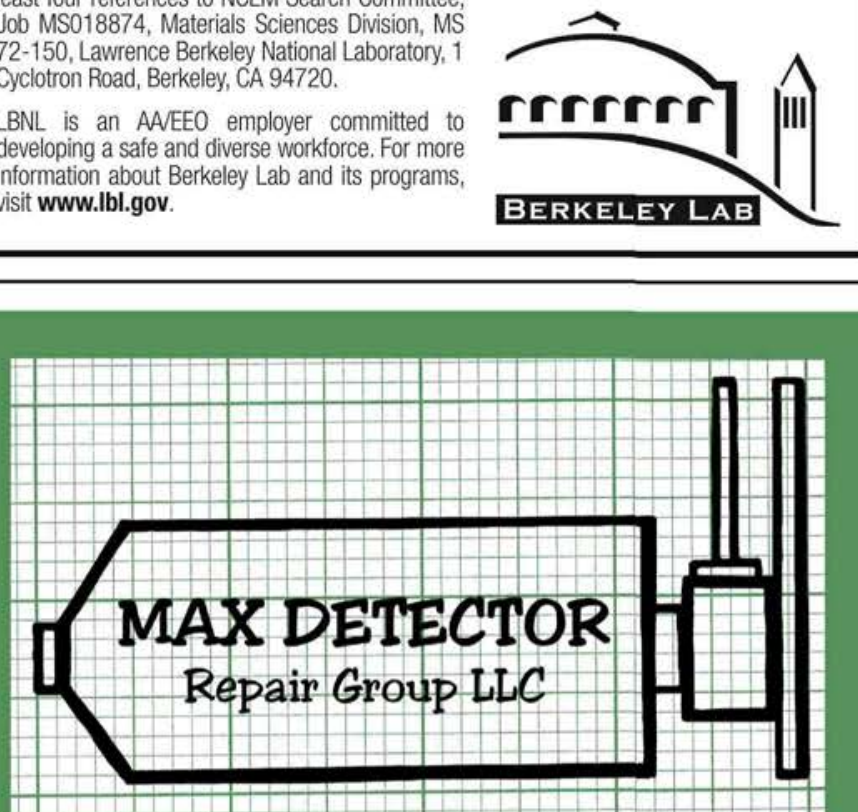

Repair and Maintenance

of all Liquid Nitrogen-Cooled Microanalysis Detectors

Central Location
Full-Service Facility
Quick Turn-Around
Experienced Personnel
Excellent Results
(608) 829-0110
WWW.MAXDETECTOR.com

\section{The Leaders in Lift Out Technology}

Now selling $5^{\text {th }}$ Generation Ex-Situ Systems

INTRODUCING THE WORLDS FIRST AUTOMATED EX-SITU LIFT OUT SYSTEM

\section{"THE AUTO LIFT"}

The new "Auto Lift" is unmatched in maximizing TEM Sample throughput. No other instrument or technique is faster when working with a FIB. Great for Clean Room Environments!

The Ex-Situ Method by Micro Optics of Florida, Inc.

- The "Original" Lift Out Technique

- The New "Auto Lift" for high

throughput.(Easy Upgrade for Existing

Customers)

- Allows your FIB to do what it does best: Make TEM Samples

- Proven, Fast, Easy Technology Over 100 sold worldwide

- Success Rate is $95 \%$

- Cost Effective Systems including: The New "Auto Lift", Standard One and Two Manipulator systems, and a University model

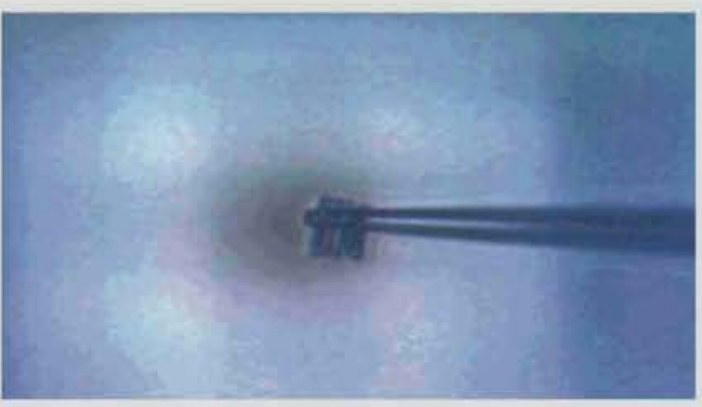

Micro Optics of Florida, Inc. (800) 545-3996 info@microopticsfl.com 


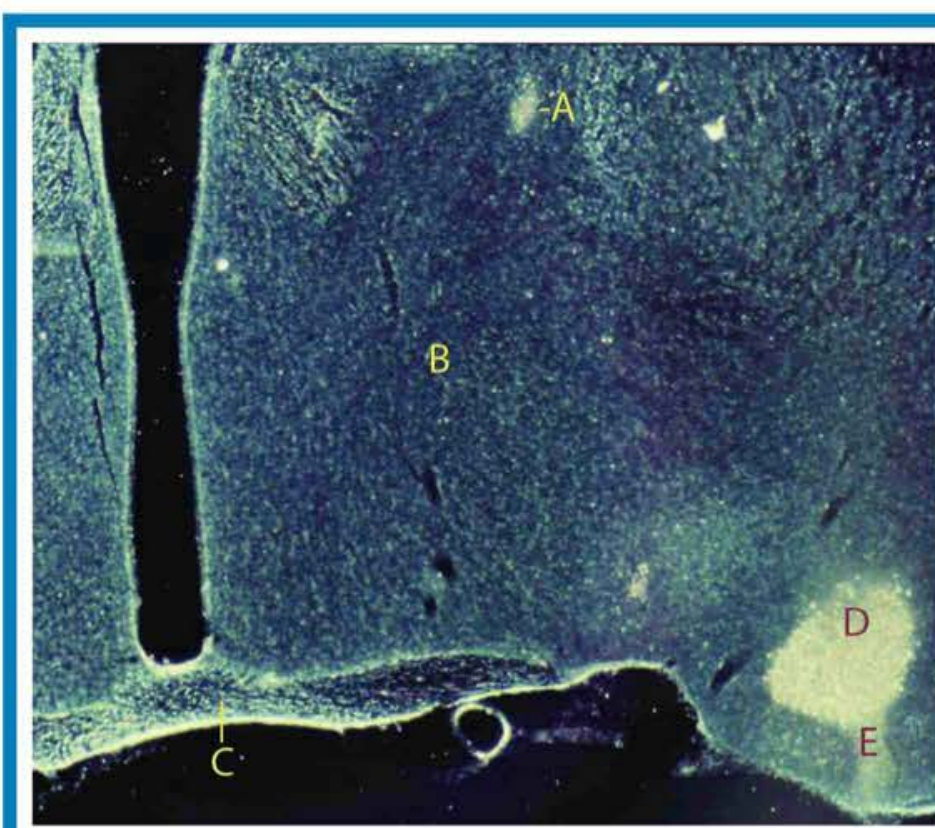

A.) Labeled fiber tract in cross section

B.) Negligible background, no red blood cells

C.) Labeled Axons Crossing the midline below the optic chiasm

D.) Dense cells, unusually intensely stained

E.) HRP reaction product in cut axons or Terminals

Figure1. Low power darkfield photomicrograph of brain section from a hamster perfused with pressurized sucrose, showing HRP labeled cells and fibers. Total magnification 14x. Note the near absence of red blood cells and negligible background staining.

properties of cells generally. Thus, the mechanism described above to result in shrinkage and distortion as a result of traditional fixation applies to all tissues. Nevertheless, avoiding shrinkage is more valuable to neuroscientists than to scientists studying most other organs. Brain has about $20 \%$ extracellular space. Other tissues likely have less, (e.g. skin) and will shrink correspondingly less. The brain is more variable in consistency, with differing densities of gray and white matter throughout. Thus, it will likely distort more as result of shrinkage than other more homogeneous organs. In brain, unlike most other tissues, localization is usually a much more important issue in histology than cell morphology, and distortion a more serious problem. Red blood cells react with or interfere with specific cell stains (HRP, immunoflurescence) very important to the neuroscientist; the high pressure of this protocol is very effective at removing red blood cells. In organs without the blood-brain barrier, plasma fluids and extracellular fluids mix more readily at physiological pressures. It would thus not be necessary to pump the pressure up to $300 \mathrm{mmHg}$, or even above physiological range, to replace the sodium ions with sucrose in organs other than the brain. However, it might still be useful to remove the red blood cells.

For effective fixation of organs, the red blood cells must be removed in order to let fixative penetrate throughout the vascular system; the high pressure of this protocol is very effective at clearing the red blood cells to allow homogeneous fixation, relevant in any tissue. Fixative must arrive as soon after anoxia begins as possible, to avoid deterioration; clearing the blood fast allows the fixative to begin to flow sooner, and with less obstruction.

\section{A Test}

Hamsters were anesthetized deeply and then perfused by inserting a large (low resistance) gavage needle through the heart into the ascending aorta, and clamping it into place. Pressure was pumped up as rapidly as possible to $300 \mathrm{~mm} \mathrm{Hg}$, over about 5 or 6 seconds (it is not desirable to pre-pressurize; since the blood should be evacuated before breaking the blood brain barrier). The animals were perfused with sucrose until muscle movement stopped, after which flow was switched to fixative and the perfusion continued with about $500 \mathrm{ml}$ of fixative at $100 \mathrm{~mm} \mathrm{Hg}$. In this case, the solution for the prewash was $10 \%$ sucrose, slightly hypertonic, and the solution for the fixative was $4 \%$ paraformaldehyde, $1 \%$ glutaraldehyde in distilled water. Although I didn't know it at the time, this second solution, although of high osmolarity, was very hypotonic, since these fixatives crossed the cell membrane. We avoided the shrinkage, but these brains may be actually enlarged and edematous. Some recent data we are collecting on this with the aid of a Plethsymometer (volume meter) shows brains perfused this way are larger than fresh tissue brains. However, the ability to get larger brains is itself promising, since calibrating the fixative solution used should get to the right sized brain.

The results were dramatically noticeable in several ways. Upon removal of the brains, their gross appearance was much larger and whiter than we were used to. Previously, brains perfused at low pressures with a saline prewash, and fixed by paraformaldehyde/glutaraldehyde fixative, had a shrunken, reddish look, and a harder consistency. With the new procedure, coronal sections of hamster brain were clearly larger and more anatomically correct. Ventricles were slits as in fresh tissue, not wide ovals as we were used to seeing. Sections would no longer fit side by side on 1 inch width slides, but had to be arranged lengthwise on the slide. Presumably, these brains were enlarged, and cells bloated. HRP reactions on this tissue had very low background, and stained cells were strongly reactive. Strong HRP reaction indicated that the tissue was indeed fixed, since underfixed tissue does not react in HRP stains. See Figure 1 and Figure 2.

Dr. Miles Cunningham used the pressure sucrose protocol but with all isotonic solutions to perfuse the rat brains shown in the attached picture, Figures 3. Note the center brain in Figure 3

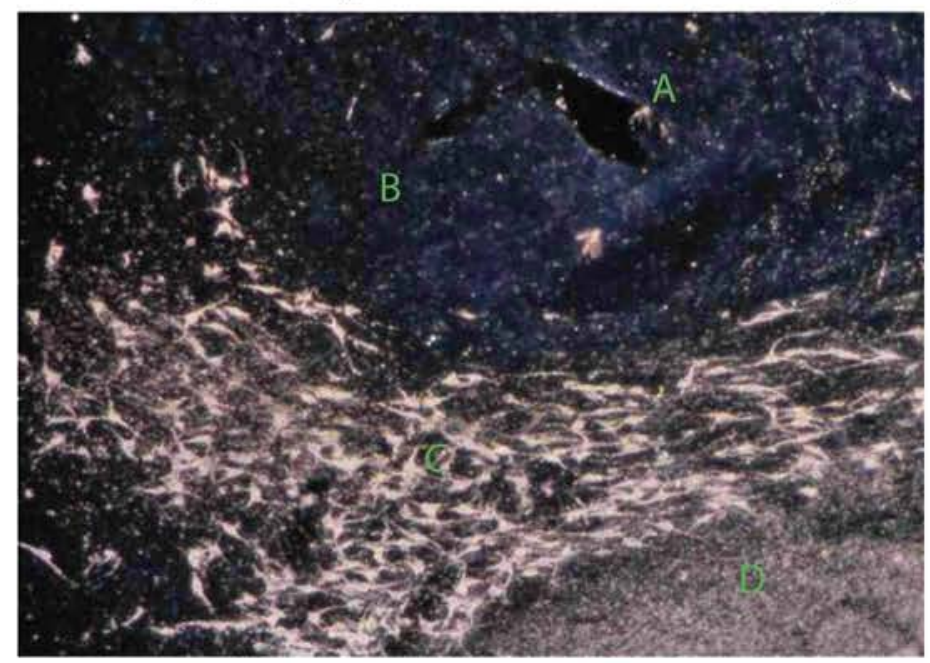

A.) Red blood cell in capillary

B.) Dark background, devoid of stain

C.) Dense, strongly labeled large cells

D.)HRP reaction product within cut axons or terminals

Figure 2. High power darkfield photomicrograph of HRP labeled cells and fibers in hamster brain perfused with pressurized sucrose. Total magnification 200x. Note the vivid staining and dark background. HRP Staining proves effective fixation, HRP reaction gets null staining in unfixed tissue. 


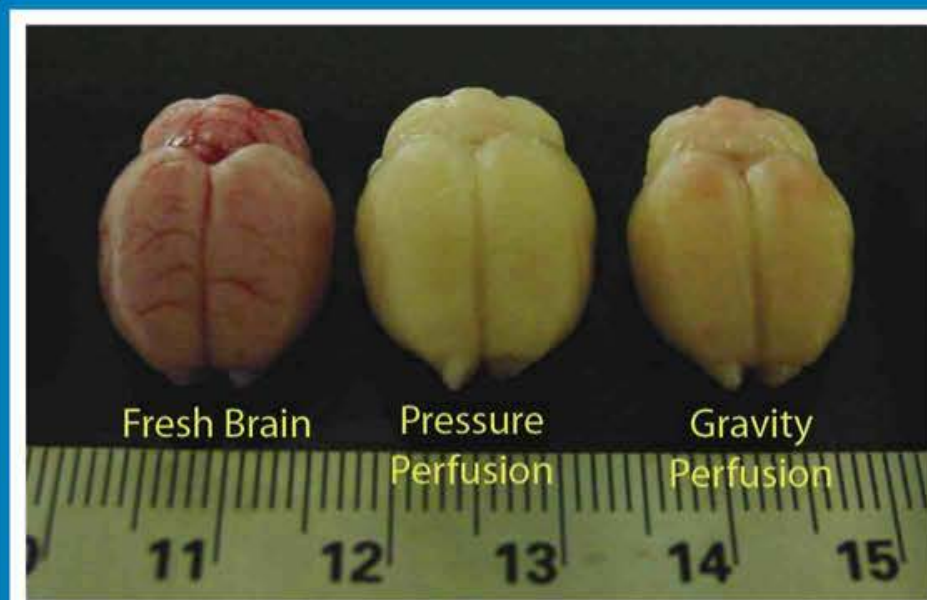

Figure 3. Perfusion by different methods

is whiter, and freer of red blood cells. The brains appear of comparable size.

We are now employing a Plethysmometer to measure the volume of brains following extraction after perfusion. Groups included pressure or gravity force, and sucrose or saline, plus fresh brains. We expect from our work to develop a perfusion solution and protocol that will avoid shrinkage of brain tissue. Critical concepts are:

1) It is necessary to avoid the sudden burst of swelling caused by sodium inrush and autolysis that accompanies most cell death events, because:

2) Once the extracellular space is closed, it cannot be reopened.

3) Reducing cellular swelling with closed extracellular spaces will shrink the whole organ.

4) Pressure isotonic sucrose, free of ions, can wash out the extracellular fluid and thus avoid sodium inrush.

5) Further, devoid of ions and continuously being refreshed, the sucrose creates a steep outward gradient for all ions in the cell, including those newly released by autolysis.

6) Some cellular shrinkage is expected toward the end of the sucrose prewash, as the ICF becomes depleted in ions and tonicity, enlarging the extracellular space, but not by whole organ shrinkage.

7) Cragg and our own work have shown that pressure isotonic sucrose is sufficient to avoid closing of the extracellular spaces, apparently staying ahead of the intracellular tonicity increase due to autolysis alone, without the sodium inrush.

8) Whether the cells are shrunken or swollen, and the size of the extracellular space, is then dependent on the changing tonicity state of the intracellular fluids relative to the fixed and flowing extracellular fluids.

9) It will be possible to develop a fixative/vehicle that leaves $20 \%$ open extracellular space and unshrunk whole organ volume. This might be hypotonic sucrose, to match the final fixed intracellular tonicity, or might include a buffer to set the $\mathrm{pH}$, and put some ions back into the intracellular fluid, at a somewhat higher tonicity.

\section{References}

Baker, J.R., Principles of Biological Microtechnique, Methuen \& Co. Ltd, 37-40, 1958

Cammermeyer, J. Post mortem origin and mechanism of neuronal hyperchromatosis and nuclear pyknosis.

Exp. Neurol. 2:379-405, 1960

Cragg, B., Preservation of extracellular space during fixation of the brain for electron microscopy. Tissue and Cell 12(1): 63-72, 1980;
Fox, C.H., et.al. Formaldehyde fixation. J Histochem. Cytochem. 33: $845-853$, 1985

Garman RH. Artifacts in routinely immersion fixed nervous tissue.

Toxicol Pathol:18, 149-531, 1990

Green, C.J.. Animal Anaesthesia. Laboratory Animal Handbooks 8. Laboratory Animals Ltd., London, UK. pp. 123-124, 1979

Helander, K.G. Formaldehyde binding in brain and kidney: A kinetic study of fixation. The Journal of Histotechnology. 22(4): 317-318, 1999

Helander, K.G., Kinetic studies of formaldehyde binding in tissue. Biotechnique and Histochemistry. 69: $177-179,1994$

Kerner M., et.al., Effect of formalin fixation and processing on immunohistochemistry. Am J Surg Pathol. 24(7): 1016 -1019, 2000.

König, J. F. R. and Klippel, R. A., The Rat Brain. A Stereotaxic Atlas of the Forebrain and Lower Parts of the Brain Stem. Robert E. Kreiger Publishing Co. Inc., Huntington, New York, 1967.

Medawar, P.B., The rate of penetration of fixatives. J R Microsc Soc. 61:, 46, 1941

Mesulam, M.M., Tetramethyl benzidene for horseradish peroxidase neurohistochemistry: a non-carcinogenic blue reaction-product with superior sensitivity for visualizing neuronal afferents and efferents. J. Histochem. Cytochem. 26: 106-117, 1978.

Paxinos G, Watson C, Pennisi M, and Topple A., Bregma, lambda and the interaural midpoint in stereotaxic surgery with rats of different sex, strain and weight. Journal of Neuroscience Methods 1: 39-43, 1985

Paxinos, G. and Watson, C. The Rat Brain in Stereotaxic Coordinates, Fourth Edition. Academic Press, New York, 1998

Rappoport, S. I. Opening of the blood-brain barrier by acute hypertension. Experimental Neurology 52: 467-479, 1976

Short, C. E. Principles \& Practice of Veterinary Anesthesia. Williams \& Wilkins, Baltimore, 1987, page 456.

Van Harreveld, A. The extracellular space in the vertebrate central nervous system. In: The Structure and Function of Nervous Tissue (ed. G. H. Bourne) Vol 4, pp 447-511 Academic Press, New York, 1972

Van Harreveld, A. and Steiner J. Extracellular space in frozen and ethanol substituted central nerouvus tissue. Anatomical Record 166 117-130, 1970.

\section{Research Assistant in Electron Microscopy}

The Burnham Institute for Medical Research (www.burnham.org) forms part of a vibrant scientific community situated next to the Pacific Ocean, including the Scripps and Salk Institutes, as well as UCSD. We are currently seeking an Electron Microscopy Research Specialist to join an exciting interdisciplinary initiative to define the molecular processes that drive cell migration.

The position is in Dr. Hanein's laboratory, which has several stateof-the art microscopes including an FEI Tecnai G2 $120 \mathrm{KeV}$, a 200 $\mathrm{KeV}$ (FEG), a Polara $300 \mathrm{KeV}$ (shared with UCSD), and will be accepting a new $300 \mathrm{KeV}$ instrument within an 18 month period. All TEMs are equipped with CCD cameras, GIF, and fully set-up for cryo-tomography.

The successful applicant will handle an individual research project in the lab and participate in development of new modes of image acquisition, train lab members and perform some microscope and ancillary equipment maintenance (fully supported by service contracts).

Requirements include a working knowledge of high resolution TEM and a Ph.D. or equivalent experience in Material Science, Biology or Bioengineering.

To apply, please send a current curriculum vitae and names of 3 references to humanresources@burnham.org. Please reference job code 151360410061 . Salary is competitive and commensurate with experience. Equal Opportunity Employer.

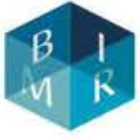
for MEDICAL RESEARCH From Research, the Pourer to Cure 